\section{THE SPECTRUM OF URANUS *}

$\mathrm{I}$ the paper "On the Spectra of some of the Fixed Stars," + presented conjointly by Dr. Miller and myself to the Royal Society in I854, we gave the results of our observations of the spectra of the planets Venus, Mars, Jupiter, and Saturn; but we found the light from Uranus and Neptune too faint to be satisfactorily examined with the spectroscope.

By means of the equatorial refractor of 15 inches aperture, by Messrs. Grubb and Son, recently placed in my hands by the Royal Society, I have succeeded in making the observations described in this paper of the remarkable spectrum afforded by the light of the planet Uranus.

It should be stated that the spectrum of Uranus was observed by Father Secchi in 1869 . He says, "Le jaune y fait complétement défaut. Dans le vert et dans le bleu il y a deux raies très larges et très noires." He represents the band in the blue as less refrangible than $F$, and the one in the green as near $\mathrm{E}$.

The spectrum of Uranus, as it appears in my instrument, is represented in the accompanying diagram. The narrow spectrum placed above that of Uranus gives the relative positions of the principal solar lines, and of the two strongest absorption-bands produced by our atmosphere, namely, the group of lines a little more refrangible than $D$, and the group which occurs about midway from $C$ to $D$. The scale placed above gives wave-lengths in millionths of a millimetre.

The spectrum of Uranus is continuous, without any part being wanting, as far as the feebleness of its light permits it to be traced, which is from $C$ to about $G$.

On account of the small amount of light received from this planet, I was not able to use a slit sufficiently narrow to bring out the Fraunhofer lines. The positions of the bands produced by planetary absorption, which are broad and strong in comparison with the solar lines, were determined by the micrometer and by direct comparison with the spectra of terrestrial substances.

The spectroscope was furnished with one prism of dense flint-glass, having a refracting angle of $60^{n}$, an observing telescope magnifying $5 \frac{1}{2}$ diameters, and a collimator of 5 inches focal length. A cylindrical lens was used to increase the breadth of the spectrum.

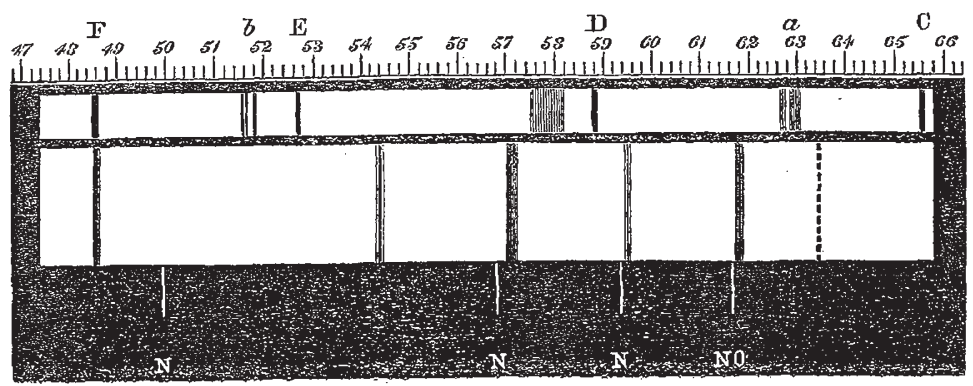

The remarkable absorption taking place at Uranus shows itself in six strong lines, which are drawn in the diagram. The least refrangible of these lines occurs in a faint part of the spectrum, and could not be measured. Its position was estimated only, and on this account it is represented in the diagram by a dotted line. The positions of the other lines were obtained by micrometrical measures on different nights. The strongest of the lines is that which has a wave length of about 544 millionths of a millimetre. The band at 572 of the scale is nearly as broad but not so dark; the one a little less refrangible than $\mathrm{D}$ is narrower than the others.

The measures taken of the most refrangible band showed that it was at, or very near, the position of $F$ in the solar spectrum. The light from a tube containing rarefied hydrogen, rendered luminous by the inductionspark, was then compared directly with that of Uranus. The band in the planet's spectrum appeared to be coincident with the bright line of hydrogen.

Three of the bands were shown by the micrometer not to differ greatly in position from some of the bright lines of the spectrum of air. A direct comparison was made when the principal bright lines were found to have the positions, relatively to the lines of planetary absorption, which are shown in the diagram. The band which has a wave-length of about 572 millionths of a millimetre is less refrangible than the double line of nitrogen which occurs near it. The two planetary bands at 595 and 618 of the scale appeared very nearly coincident with bright

* From the Proceedings of the Royal Society.

7 Phil. Trans. 1864, P. 413 ; and for Mars, Monthly Notices R. Ast. Soc. vol. $x \times$ vii., p. 178 .

\pm Comptes Rendus, vol, Ixyiii, p, 76r, and "Le Soleil," Paris, 1870, p. 354. lines of air. The faintness of the planet's spectrum did not admit of certainty on this point; I suspected that the planetary lines are in a small degree less refrangible. There is no strong line in the spectrum of Uranus in the position of the strongest of the lines of air, namely, the double line of nitrogen.

As carbonic acid gas might be considered, without much improbability, to be a constituent of the atmosphere of Uranus, I took measures with the same spectroscope of the principal group of bright lines which present themselves when the induction-spark is passed through this gas. The result was to show that the bands of Uranus cannot be ascribed to the absorption of this gas.

There is no absorption-band at the position of the line of sodium. It will be seen by a reference to the diagram that there are no lines in the spectrum of Uranus at the positions of the principal groups produced by the absorp. tion of the earth's atmosphere.

\section{WILLIAM HUGGINS}

\section{NOTES}

WE understand that the contributors to the next volume of the Zoological Record are as follows:--Mammalia, Reptilia, and Pisces, Dr. Albert Günther, F.R.S. ; Aves, Mr. H. E. Dresser, F.Z.S., and Mr.R. B. Sharpe,F.L.S. ; Mollusca, Molluscoidea, and Crustacea, Dr. Edward von Martens, F.M.Z.S. ; Arachnida and Myriapoda, Mr. O. Pickard-Cambridge, C.M.Z.S. ; Insecta generally and Culeoptera, Mr. E. C. Rye; Lepidoptera, Mr. W. F. Kirby; Diptera, G. A. Verrall; Neuroptera and Orthoptera, Mr. M'Lachlan ; Rhynchota, Mr. John Scott ; Vermes, Mr. E. Ray Lankester ; Echinodermata, Colenterata, and Pro- 